\title{
Developing Operating Procedures For Projects Involving Multiple Organizations Using a Linear Responsibility Chart
}

\author{
By Tom Clark Project Success Incorporated \\ www.projectsuccess.com
}

One of the difficulties of managing projects that involve several (perhaps many) organizations is that the group has no pre-established procedures for handling actions that cross organizational boundaries. Such actions often include:

- Technical decisions (e.g., specification or design changes)

- Managerial decisions (e.g., schedule changes)

- Administrative processes (e.g., issuing payments for work)

- Project activities that involve more than one organization (e.g., approvals or inspections, placing purchase orders)

If such inter-organizational actions are not anticipated and procedures put in place to guide their performance, confusion and miscommunication will result, which will lead to unnecessary delays, wasted resources, and potentially even conflict among the organizations.

The development of operating procedures for multi-organizational projects can be facilitated by the use of a tool known as a "liner responsibility chart" (LRC). Consider the hypothetical and simplified example illustrated on the next page. Cavendish Chemicals is planning the design and construction of their new Plant Clearwater. The project will involve the individuals, departments, and organizations shown in the columns of the chart. The inter-organizational actions that can be anticipated on this project are listed in the rows of the chart. Several responsibility codes (letters) are defined in the upper left corner, and these codes are used in each cell of the chart to indicate the responsibility(s) of the entity in that column relative to the action in that row. By reading the codes in any row of the LRC, it is possible to ascertain an overview of the procedure for the action associated with that row.

The LRC, however, is not intended to be an end in itself. Rather, the LRC is an efficient tool that is used to collect and verify information about how the organizations intend to work together, so that written procedures for each action can be developed quickly and with minimum rework. The process involves several steps as follows:

1. Identify the individuals, departments, and organizations that should be represented in the chart and develop the column headings for those entities.

2. Develop an initial set of responsibility codes, such as the codes shown in the example.

3. Interview the project manager. Make an audio recording of the interview. Ask the project manager to:

- Identify inter-organizational actions that should be included in the chart and ultimately in the project procedures manual. Enter these actions in the rows of the chart. 
- Talk through the procedure for each action as he or she would prefer that it be performed. Enter responsibility codes into the cells of the chart to capture the procedure as described. If necessary, create additional codes. 


\section{Cavendish Chemicals, Inc.}

\section{Linear Responsibility Chart}

For

\section{Design and Construction of Plant Clearwater}

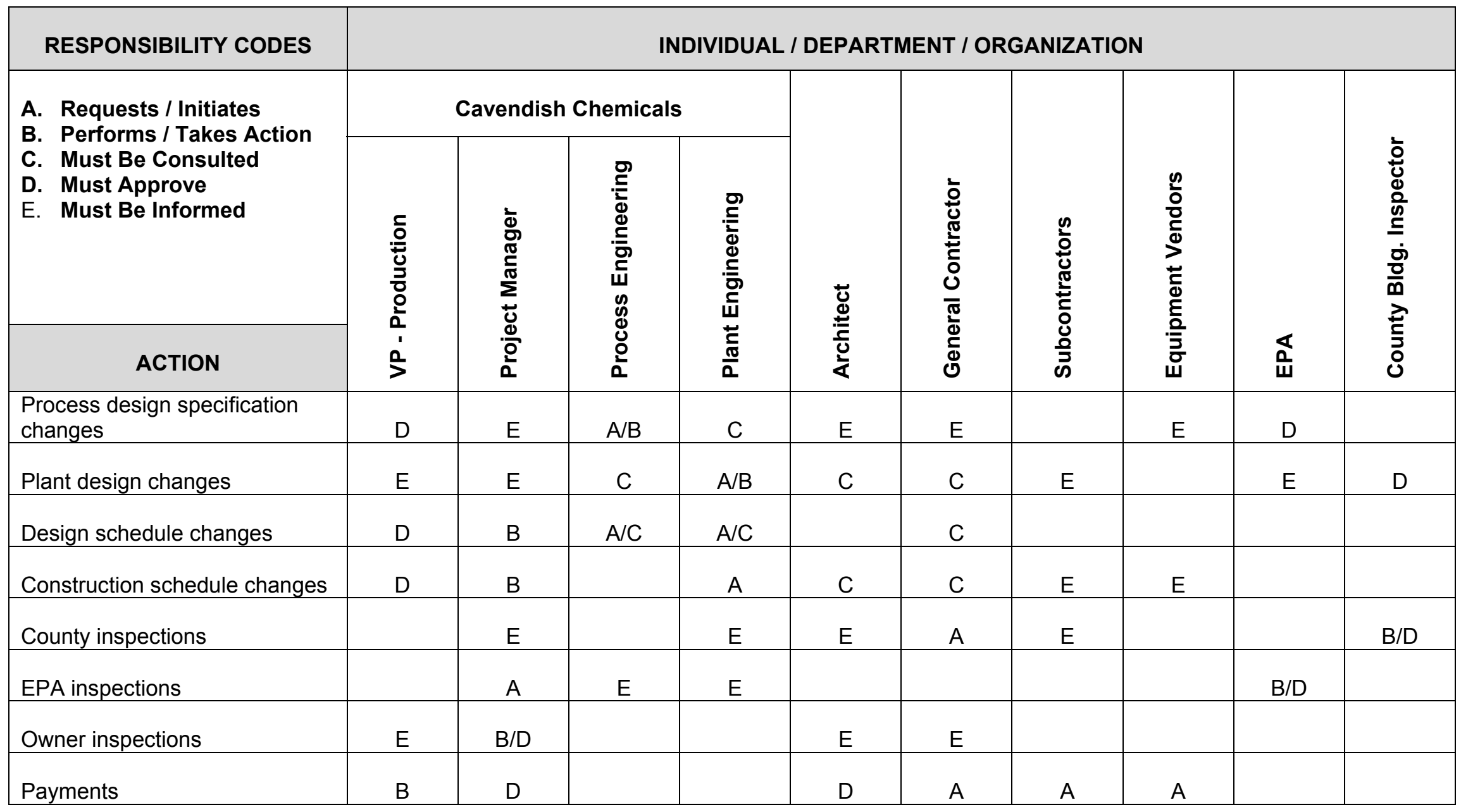


4. Interview other key individuals who are identified in the LRC or who represent departments or organizations identified in the chart. The order in which these interviews are conducted is not critical. Show each interviewee the actions that have been entered into the LRC by previous interviewees. Ask the interviewee to identify additional actions that should be included and to describe the preferred procedure. Again, enter the appropriate codes in the LRC, create additional codes if necessary, and make an audio recording of the interview.

5. When the interviews have been completed, hold a meeting of the key individuals who are identified in the LRC or who represent departments or organizations identified in the LRC. Make an audio recording of the meeting. Give out copies of the LRC.

- Explain the procedure for each action as described by the responsibility codes. Ask for comments, suggestions, or concerns on the procedure for each action. Seek consensus on all changes. Make changes to the codes as appropriate.

- Ask for any additional actions that should be added to the LRC. Have the group discuss the preferred procedure for any such actions and record the appropriate codes on the LRC. Again, seek consensus.

6. Using the LRC (and audio recordings as necessary), develop a project procedures manual. Each row on the LRC should be converted to a written procedure. Each procedure should have:

a. Date and draft number

b. Action name/description (e.g., "Construction schedule changes")

c. Statement of the procedure based on the codes in the LRC. In addition, the statement can contain details, such as:

- When making a submittal, exactly what documentation to provide and to whom it should be sent.

- How long an entity normally has to review and act on an item submitted for their approval.

- Who should receive copies of certain communications.

- Whether hard copy or electronic communication are required/allowed.

d. Signature lines for the project manager and for other key individuals who are identified in the LRC or who represent departments or organizations identified in the LRC. The signatures indicate that these key individuals approve the procedure and that they will follow and require other members of their organization follow the procedure.

In addition to the individual procedures, the procedures manual should contain:

- A table of contents

- A directory of key individuals involved in the project, including phone numbers, mailing

- addresses, and email addresses

- The final LRC on which the procedures are based

- A glossary of terms, if necessary

7. Distribute the procedures manual to all involved organizations in hard copy and/or electronic format.

The early development of a procedures manual as described above has proven invaluable on projects involving multiple organizations. The application of the LRC in the context of the steps 
outlined above greatly facilitates the process, and it ensures that the procedures manual is complete and represents consensus among the involved organizations.

\section{About the Author, the Project Success Method \& Project Success Inc} Thomas B. (Tom) Clark, Ph.D.

Co-founder and former Executive Vice President

Tom is heavily involved in the development and delivery of PSl's courses. In addition to his work with PSI, he is Professor Emeritus of Management at Georgia State University. He also served the University as Chair of the Department of Management and as Interim Dean of the College of Business Administration. Previously, he was an Assistant Professor of Industrial and Systems Engineering at Georgia Tech.

Tom has provided project management consulting and training services for a variety of business, government, and non-profit organizations. He developed and marketed one of the first PC-based software tools for project scheduling and cost control. Prior to beginning his academic career, Tom served in the U.S. Army Management Systems Support Agency at the Pentagon and was employed as an industrial engineer with a national firm in the printing industry. He holds bachelors and masters degrees in Industrial Engineering from Georgia Tech and a Ph.D. in Business Administration from Georgia State University. Tom has received several awards for teaching excellence and public service.

Since 1983, Atlanta, GA based Project Success Incorporated (formerly YCA) has been providing Project Success Method project management training and consulting services to hundreds of successful organizations, including many of the largest and most profitable Fortune 500 companies.

The Project Success clearly defines a proven approach to superior project performance. While an estimated $70 \%$ to $90 \%$ of corporate strategic initiatives fail, companies that employ The Project Success Method $®$ consistently plan and control their projects successfully, delivering projects faster, more effectively, and at lower cost.

The Project Success Method is a blueprint for planning and controlling projects of all sizes and provides the missing link between strategy formulation and implementation. The methodology is not software-specific and requires no prior project management training or professional certification. Most importantly, the Project Success MethodSM is easy to learn, highly effective and can be implemented quickly in as little as 5 days."

PSI consultants have been engaged in more than 10,000 projects in 25 countries on six continents. For more information contact walter.urban@projectsuccess.com or visit www.projectsuccess.com. 